\title{
Iperparatiroidismo primitivo in gravidanza: conseguenze materno-fetali
}

\author{
Gianlorenzo Dionigi ${ }^{1} \cdot$ Paolo del Rio ${ }^{2}$ \\ Accettato: 11 maggio 2020 \\ (c) Springer Nature Switzerland AG
}

Commento a:

Primary hyperparathyroidism in pregnancy: maternofetal outcomes at a quaternary referral obstetric hospital, 2000 through 2015.

\section{J. Rigg, E. Gilbertson, H.L. Barrett, F.L. Britten, K. Lust.}

\section{J Clin Endocrinol Metab (2019) 104:721-729}

Un iperparatiroidismo primario (IP) è stato osservato in circa l'1,4\% delle gravidanze [1]. Poiché sono riportate sia complicanze materne (pre-eclampsia; iperemesi gravidica; crisi ipercalcemica) sia fetali (ipocalcemia neonatale con tetania; aborto spontaneo; ritardo della crescita intrauterina; riduzione del peso alla nascita), la decisione in merito a terapia farmacologica $v s$ chirurgia è complessa [1]. In generale, si può presumere che la concentrazione sierica materna di calcio aumenti il rischio di complicanze [1]. Tuttavia, non esiste ancora un consenso sui valori limite di calcio, per favorire una decisione per la procedura chirurgica o per la terapia medica [1].

Un recente studio retrospettivo riporta 28 pazienti con IP durante la gravidanza, 22 sono stati trattati con terapia farmacologica, 6 con chirurgia presso l'Ospedale australiano di Brisbane in un periodo di 15 anni [2]. Sono stati analizzati tutti i dati disponibili sulle analisi di laboratorio, il decorso della gravidanza, la nascita e l'esito dopo la terapia farmacologica o chirurgica del IP [2]. Dal momento in cui è stato diagnosticato il IP, che avrebbe potuto influenzare la decisione

$\triangle$ G. Dionigi

gdionigi@unime.it

1 Divisione di Chirurgia Endocrina e Mininvasiva, Dipartimento di Patologia umana dell'adulto e dell'età evolutiva, Università di Messina, Messina, Italia

2 Divisione di Chirurgia Generale, Dipartimento di Medicina e Chirurgia, Università di Parma, Parma, Italia terapeutica per una procedura operativa, sono stati formati 2 gruppi: terapia farmacologica prima o dopo la $28^{\mathrm{a}}$ settimana di gravidanza, e terapia chirurgica nel $2^{\circ}$ trimestre. Il range di età delle donne andava da 30 a 36 anni [2]. I valori sierici iniziali nei gruppi erano 2,7-2,9 e 2,8-3,1 mmol/L. Le 6 pazienti con paratiroidectomia presentavano un adenoma con normalizzazione postoperatoria del calcio e dell'ormone paratiroideo. La gestazione media al momento dell'intervento è stata di 24 settimane (intervallo, da 18 a 32). Tre delle quattro pazienti sottoposte a chirurgia mininvasiva avevano identificato lesioni target durante l'imaging, mentre l'imaging non era determinante per coloro che erano state sottoposte a esplorazione bilaterale. L'istologia ha confermato la presenza di singoli adenomi delle paratiroidi in quattro pazienti, una paziente aveva tessuto paratiroideo iperplastico e un'altra aveva un possibile adenoma paratiroideo. Sono state richieste cure mediche in tre delle sei gravidanze gestite chirurgicamente e queste pazienti avevano concentrazioni sieriche di calcio $3 \mathrm{mmol} / \mathrm{L}$. La terapia più comune utilizzata era l'idratazione. Una paziente ha ricevuto fosfato e cinacalcet senza esiti avversi. Una paziente ha ricevuto farmaci antiipertensivi per l'ipertensione indotta dalla gravidanza [2]. Le seguenti complicanze si sono verificate nelle pazienti trattate con farmaci: pre-eclampsia $(6 / 22 ; 30 \%)$; ipertensione in gravidanza $(3 / 22 ; 15 \%)$; parto prematuro dovuto a pre-eclampsia $(4 / 22 ; 20 \%)$; aborto spontaneo $(2 / 22$; $10 \%)$; per le pazienti trattate chirurgicamente: nessuna preeclampsia, 2 casi di ipertensione in gravidanza, nessun caso di parto prematuro [2]. Neonati: $4 / 22$ bambini delle pazienti trattate con farmaci necessitavano di terapia intensiva dopo il parto prematuro, uno di questi bambini presentava lieve ipocalcemia. Dopo la paratiroidectomia, un bambino nato ha dovuto essere sottoposto a trattamento intensivo per ipertensione polmonare. Non sono state registrate complicazioni intra- e postoperatorie [2]. 
Il vantaggio dello studio è l'attenta elaborazione dei dati clinici delle pazienti e la revisione della letteratura. Il limite del lavoro è il numero limitato di casi [1, 2]. I protocolli di gestione dell'anestesia non sono stati riferiti nello studio. L'attuale studio manca di dati sulle IP geneticamente determinate [2].

Tuttavia, sulla base dei risultati disponibili dello studio e dell' analisi della letteratura, il IP in gravidanza è associato a un'alta incidenza di complicanze fetali e neonatali associate direttamente proporzionali al grado dei livelli sierici di calcio materno. Il rischio di pre-eclampsia durante sola terapia farmacologica è aumentato rispetto alla terapia chirurgica, in particolare con valori di calcio $>3 \mathrm{mmol} / \mathrm{L}[1,2]$. Considerando il rischio complessivo di un intervento chirurgico durante la gravidanza (significativamente inferiore rispetto al passato), i dati dello studio suggeriscono l'intervento chi- rurgico con IP, in particolare con valori di calcio sierico crescenti $>3 \mathrm{mmol} / \mathrm{L}[1,2]$. La gestione chirurgica, quando necessario, viene idealmente eseguita nel secondo trimestre.

Nota della casa editrice Springer Nature rimane neutrale in riguardo alle rivendicazioni giurisdizionali nelle mappe pubblicate e nelle affiliazioni istituzionali.

\section{Bibliografia}

1. Dralle H, Weber T (2020) Parathyroidectomy during pregnancy. Chirurg. https://doi.org/10.1007/s00104-020-01128-4

2. Rigg J, Gilbertson E, Barrett HL et al (2019) Primary hyperparathyroidism in pregnancy: maternofetal outcomes at a quaternary referral obstetric hospital, 2000 through 2015. J Clin Endocrinol Metab 104(3):721-729 